\title{
Persistent currents in toroidal dipolar supersolids
}

\author{
M. Nilsson Tengstrand, ${ }^{*}$ D. Boholm, R. Sachdeva, J. Bengtsson $\odot$, and S. M. Reimann $\odot$ \\ Mathematical Physics and NanoLund, Lund University, Box 118, 22100 Lund, Sweden
}

(Received 24 March 2020; revised 5 October 2020; accepted 7 December 2020; published 15 January 2021)

\begin{abstract}
We investigate the rotational properties of a dipolar Bose-Einstein condensate trapped in a toroidal geometry. Studying the ground states in the rotating frame and at fixed angular momenta, we observe that the condensate acts in distinctly different ways depending on whether it is in the superfluid or in the supersolid phase. We find that intriguingly, the toroidal dipolar condensate can support a supersolid persistent current which occurs at a local minimum in the ground state energy as a function of angular momentum, where the state has a vortex solution in the superfluid component of the condensate. The decay of this state is prevented by a barrier that in part consists of states where a fraction of the condensate mimics solid-body rotation in a direction opposite to that of the vortex. Furthermore, we find that superfluid condensates may enter the supersolid phase when forced to take angular momentum. Last, the rotating toroidal supersolid shows hysteretic behavior that is qualitatively different depending on the superfluid fraction of the condensate.
\end{abstract}

DOI: 10.1103/PhysRevA.103.013313

\section{INTRODUCTION}

In a supersolid quantum many-body state, off-diagonal and diagonal long-range order occur simultaneously [1-5]. Supersolidity has long been debated [6-9], particularly for ${ }^{4} \mathrm{He}$ with the conclusion that its observation is not supported [10]. Ultracold atomic Bose-Einstein condensates (BECs) are alternative candidates, for which supersolidity was proposed for BECs with soft-core two-body potentials [11-14], and experimentally realized using condensates coupled to two optical cavities [15]. Likewise, spin-orbit coupled BECs [16,17] have a modulated density stripe phase showing supersolid properties [18]. Quantum gases with long-range dipolar interactions (see Refs. [19,20] for reviews) are another example: For the dipolar BEC, phase transitions from a superfluid to a density-modulated supersolid and a droplet crystal phase were recently observed [21-27]. The discovery of long-lived droplets came off as imperative for the realization of supersolidity in these condensates. In dysprosium and erbium condensates, a transition from a superfluid to a metastable state was observed where mutually repulsive droplets form [28-32], each consisting of many atoms. Inducing droplets and subsequently melting them back into a BEC by Feshbachcontrolling the short-ranged (contact) part of the atom-atom interactions, a bistability in the gas-droplet transition occurs [28]. As dipoles repel each other when side-by-side but become attractive when head-to-tail, the droplets have

\footnotetext{
*mikael.nilsson_tengstrand@matfys.lth.se
}

Published by the American Physical Society under the terms of the Creative Commons Attribution 4.0 International license. Further distribution of this work must maintain attribution to the author(s) and the published article's title, journal citation, and DOI. Funded by Bibsam. spheroidal shapes being elongated along the dipolar polarization direction [30]. The physical origin of the self-binding mechanism for dipolar droplets [33-36] is similar to that in binary Bose gases [37-39] realized with potassium in two hyperfine states $[40,41]$. In either case, the stability of the droplets and their critical size is controlled by the balance between a residual mean-field interaction and quantum fluctuation contributions to the total energy, often referred to as the Lee-Huang-Yang corrections [42,43]. For the dipolar $\mathrm{BEC}$, increasing the ratio between the dipolar and short-range interaction strengths beyond a critical value, the dispersion relation shows the softening of the roton spectrum at a finite momentum $[44,45]$. With further increase of this ratio, there is a transition from the density modulated supersolid to the droplet crystal phase, where the individual droplets possess superfluid character but have no superfluidity and, hence, no phase coherence between neighboring droplets. This scenario was also confirmed by recent quantum Monte Carlo studies [46]. Evidence came from the Higgs amplitude and Nambu-Goldstone modes depicting the spontaneous symmetry breaking in the supersolid phase [24-27,47].

One of the most distinct features of superfluidity is the quantization of circulation and formation of vortices in response to rotation. The nonclassical rotational moment of inertia quantitatively differentiates the supersolid from the superfluid phase [48]. The interplay between droplet formation and vortices in rotating pancake-shaped traps was analyzed in Refs. [49], and characteristic interference patterns of rotating supersolids were found [50]. Ring-shaped traps confining a superfluid may support a persistent current [51]. This has been extensively studied theoretically for bosons with contact interactions (see, e.g., Refs. [52-57]), with optical lattices [58,59], or for dipolar gases [60-65]. For soft-core bosons, metastable superflow was also found in the supersolid phase [66]. Experiments measuring phase slips and persistent currents with ${ }^{23} \mathrm{Na}$ [67-69] envisioned "atomtronic" applications [70]. 
In this paper, we show that when a dipolar gas of dysprosium in a torus confinement is set rotating, it acts in a distinctly different way depending on whether it is in the superfluid or in the supersolid droplet phase, yet exhibiting some surprisingly general features largely independent of system parameters. When there is a persistent current in a superfluid ring trap, at $L / N \hbar=1$ (where $L$ is the angular momentum and $N$ the number of bosons) the energy as a function of angular momentum has a characteristic sharp v-shaped minimum with negative curvature [51-53], protecting the state from its dissipative decay to a nonrotating state by an energy barrier. In the supersolid regime, likewise there is a local energy minimum, however, now at $L / N \hbar<1$ and with positive curvature. In this case, the state carries a vortex in the superfluid component of the condensate. We show that the decay of this state is prevented by a barrier that in part consists of states where a component of the BEC mimics solid-body rotation in a direction opposite to that of the vortex. Furthermore, we find that for certain parameter values the ground state of the BEC may alternate between the superfluid and the supersolid phase as a function of angular momentum. When the supersolid is set rotating, it shows hysteretic behavior that is qualitatively different for different values of the superfluid fraction of the condensate. We find that a toroidal confinement is a particularly favorable setup, since the confinement inhibits the lateral repulsion between the droplets, otherwise diminishing the interdroplet tunneling [32].

\section{MODEL}

The dipolar condensate at zero temperature is modeled by a nonlocal extended Gross-Pitaevskii equation

$$
i \hbar \frac{\partial}{\partial t} \psi(\mathbf{r}, t)=\left[-\frac{\hbar^{2} \nabla^{2}}{2 m}+V_{\text {trap }}(\mathbf{r})+g|\psi(\mathbf{r}, t)|^{2}+\int d \mathbf{r}^{\prime} V_{\mathrm{dd}}\left(\mathbf{r}-\mathbf{r}^{\prime}\right)\left|\psi\left(\mathbf{r}^{\prime}, t\right)\right|^{2}+\gamma|\psi(\mathbf{r}, t)|^{3}\right] \psi(\mathbf{r}, t),
$$

where $\psi$ is the order parameter normalized to the particle number $N$, with trapping potential in cylindrical coordinates $V_{\text {trap }}(\mathbf{r})=m \omega^{2} / 2\left[\left(\rho-\rho_{0}\right)^{2}+\lambda^{2} z^{2}\right]$, where $\rho_{0}$ is the radius of the ring, $\omega$ the axial trapping frequency, and $\lambda$ the ratio of transversal and axial trapping frequencies. The contact interaction coupling constant is $g=4 \pi \hbar^{2} a / m$, where $a$ is the $s$-wave scattering length. The ratio between the dipole and contact interaction strengths is quantified by the dimensionless parameter $\varepsilon_{\mathrm{dd}}=a_{\mathrm{dd}} / a$, where $a_{\mathrm{dd}}=$ $m \mu_{0} \mu^{2} / 12 \pi \hbar^{2}$ is the dipolar length, $\mu$ is the magnetic moment, and $\mu_{0}$ the permeability in vacuum. The dipolar potential is $V_{\mathrm{dd}}(\mathbf{r})=\frac{\mu_{0} \mu^{2}}{4 \pi} \frac{\left(1-3 \cos ^{2} \theta\right)}{\mid \mathbf{r}^{3}}$, where $\theta$ is the angle between $\mathbf{r}$ and the alignment direction of the dipoles, here taken to be the $z$ direction. The last term includes quantum fluctuations in the local density approximation [35,43], with $\gamma=\frac{128 \sqrt{\pi}}{3} \frac{\hbar^{2} a^{5 / 2}}{m}\left(1+\frac{3}{2} \varepsilon_{\mathrm{dd}}^{2}\right)$. The validity of this term has been discussed in Refs. [33,35,71]. Apart from requiring the local density approximation, the condensate depletion has to be small $[33,43]$, which for the data shown in this paper is $\lesssim 0.06$.

\section{NUMERICAL RESULTS}

The ground state is found by solving Eq. (1) in imaginary time using the split-step Fourier method. In order to study the system in a rotating frame the term $-\Omega L_{z} \psi$ (where $L_{z}=x p_{y}-y p_{x}$ and $\Omega$ is the rotation frequency) is added to the right-hand side of Eq. (1), which is then solved in a similar manner. To obtain the ground state at a fixed angular momentum $L_{0}$, we instead minimize the quantity $\tilde{E}=$ $E+C \omega\left(L-L_{0}\right)^{2}$, where $E$ is the energy corresponding to Eq. (1), $L=\int d \mathbf{r} \psi^{*} L_{z} \psi$ and $C$ a dimensionless positive number that, when large enough, causes the energetic minimum to occur at $L \approx L_{0}$. (We note here that the numerical solution of Eq. (1) is involved due to many close-lying local minima in the energy surface, as commonly encountered in similar studies [36,49,50,72]. This may lead to convergence into metastable solutions, and we therefore perform an extensive sampling over a large number of different initial conditions in order to find the most probable lowest-energy solutions).

We exemplify our findings here for a system of ${ }^{164} \mathrm{Dy}$ atoms with $N=10^{4}$ in a trap where $\rho_{0}=1 \mu \mathrm{m}, \omega / 2 \pi=$ $1 \mathrm{kHz}$, and $\lambda=1.7$. Different phases of the BEC are identified by the superfluid fraction, defined through its nonclassical rotational inertia according to $f_{s}=1-I / I_{\mathrm{cl}}[5,48]$, where $I_{\mathrm{cl}}=$ $m\left\langle\rho^{2}\right\rangle$ is the classical moment of inertia and $I=\lim _{\Omega \rightarrow 0} L / \Omega$.

The numerically calculated superfluid fraction as a function of $\varepsilon_{\mathrm{dd}}$ is shown in Fig. 1. It is seen that for some

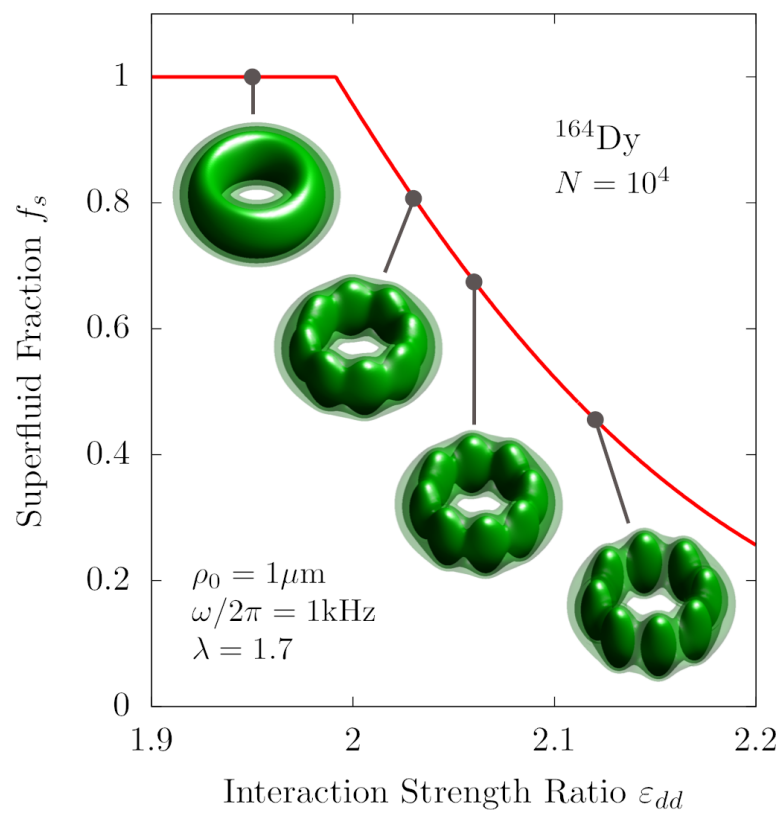

FIG. 1. Superfluid fraction $f_{s}$ as a function of the interaction strengths ratio given by the dimensionless parameter $\varepsilon_{\mathrm{dd}}$. The insets (left to right) show density isosurfaces, taken at density values $2 \times 10^{-4}, 0.5 \times 10^{-4}$, and $1.0 \times 10^{-5}$ (in units of $a_{\mathrm{dd}}^{-3}$ ), at $\varepsilon_{\mathrm{dd}}=1.95,2.03,2.06$, and 2.12 (marked by bullets) where $f_{s}=$ $1.0,0.81,0.67$, and 0.46 . 
critical value of $\varepsilon_{\text {dd }}$ the superfluid fraction $f_{s}$ drops from unity in the superfluid case to smaller values $f_{s}<1$. The insets show some typical density isosurfaces. When superfluid they are azimuthally symmetric (see the top inset in Fig. 1 for $\varepsilon=1.95$ ). The drop in $f_{s}$ is accompanied by an onset of localization (middle insets for $\varepsilon=2.03$ and 2.06), where the dipolar gas in the toroidal confinement begins to form a modulated density distribution as a precursor to droplet formation, similar to what was found in a tube with periodic boundary conditions [72,73]. As the superfluid fraction decreases the density modulation becomes more pronounced, leading to the characteristic form of spheroidal droplet-like formations $[21,30,32]$ elongated along the polarization axis of the dipolar gas and submerged into a finite background of superfluid density, indicating the coexistence of off-diagonal and diagonal long-range order. These droplets are clearly visible in the isosurfaces (see bottom right inset for $\varepsilon_{\mathrm{dd}}=2.12$ ). Let us now determine the ground state energy as a function of angular momentum, $E(L)$, for different values of $\varepsilon_{\mathrm{dd}}$; see Fig. 2. In the superfluid phase the energy has a $\mathrm{v}$-shaped local minimum at $L / N \hbar=1$. The dark-red line (dark-gray line first from top) in Fig. 2 shows $E(L)$ for $\varepsilon_{\mathrm{dd}}=1.90$. Upon increasing $L / N \hbar$ from the nonrotating state, a vortex enters in the usual way [74] and becomes localized at the center when $L / N \hbar=1$, accompanied by a phase jump of $2 \pi$ [51]. Closer to the critical value of $\varepsilon_{\mathrm{dd}}$, when approaching the supersolid regime, as here shown for $\varepsilon_{\mathrm{dd}}=1.95$ (orange/gray line second from top), the entry of the vortex becomes qualitatively different. We can see from the density isosurfaces shown in the upper panel that for angular momenta either close to the nonrotating state, or the state with one vortex, the ground state shows a supersolid-like density modulation, as visible in the plots for $L / N \hbar=0.2$ and $L / N \hbar=0.85$. For these values it is energetically favorable to add energy in terms of solid-body rotation instead of the usual vortex formation (we note that this vortex entry is accompanied by density modulations; see the isosurface for $L / N \hbar=0.3$ )

For increased $\varepsilon_{\mathrm{dd}}$, the condensate enters the supersolid phase, as we have seen in Fig. 1. Curiously, when the system becomes supersolid, a distinct local minimum in $E(L)$ for nonzero angular momentum remains, but now at a value $L / N \hbar<1$ and with a positive curvature. As in the superfluid case, this minimum is protected by an energy barrier against dissipative decay to the nonrotating state. The insets at the bottom in the lower panel of Fig. 2 show the phase and density contours on either side of the energy barrier, at $L / N \hbar=0.3$ and at the position of the minimum at $L / N \hbar=0.74$. For reference, we also include the phase and density contour in the superfluid phase for $\varepsilon_{\mathrm{dd}}=1.95$ at $L / N \hbar=1.0$; see the inset at the top of the lower panel in Fig. 2.

While for lower angular momenta the phase shifts indicate solid-body rotation, at the minimum beyond the energy barrier the system has developed a singly-quantized vortex. The typical phase jump of $2 \pi$ occurs simultaneously with the dipolar density modulation. The density contours (white lines) well reflect the density isosurfaces for the corresponding values of $\varepsilon_{\mathrm{dd}}$ shown in Fig. 1. By increasing $\varepsilon_{\mathrm{dd}}$ further towards the limit of droplet localization, the local energy minimum with positive curvature eventually disappears, and the energy instead becomes a monotonically increasing function, no longer

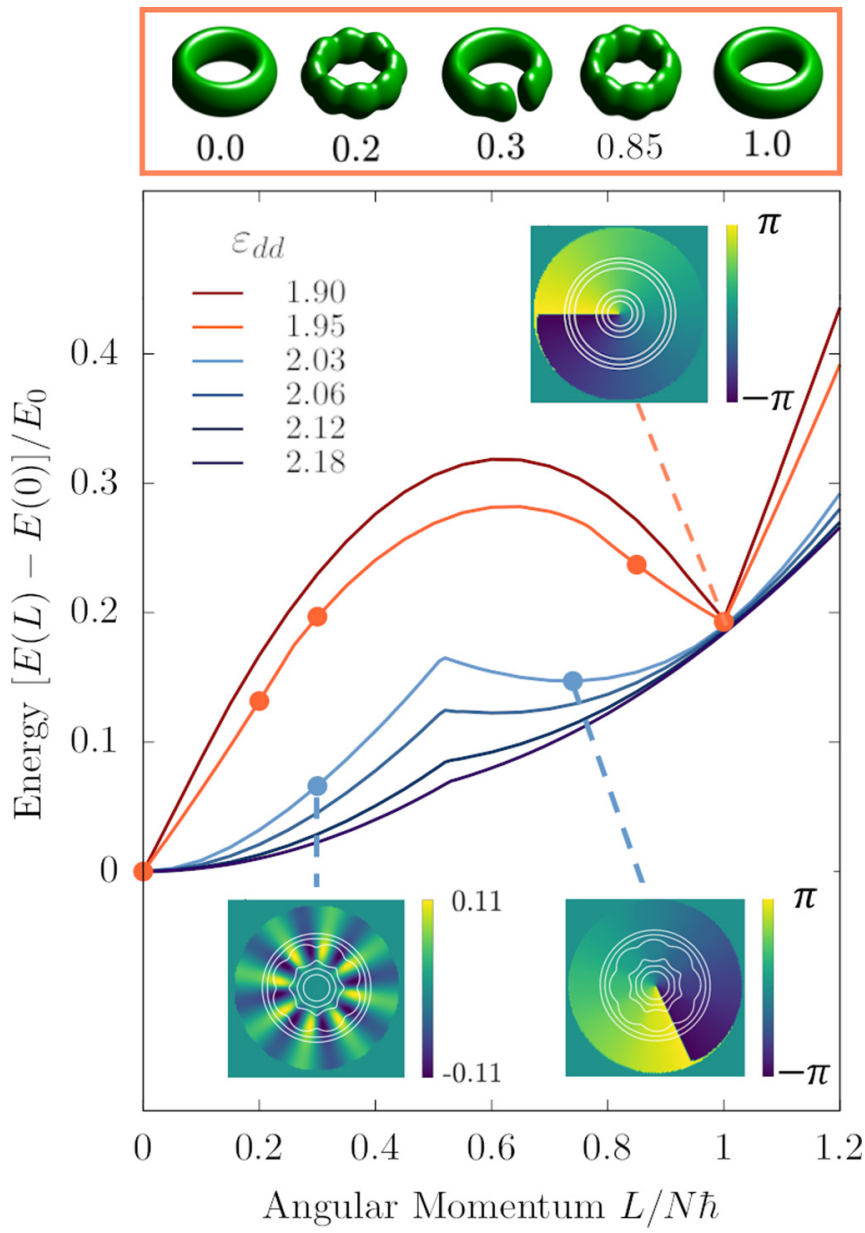

FIG. 2. Upper panel: Density isosurfaces for $\varepsilon_{\mathrm{dd}}=1.95$ (taken at the density value $2 \times 10^{-4} a_{\mathrm{dd}}^{-3}$ ), at angular momenta as specified by the numbers given (also indicated by the bullets on the second line from the top in the lower panel). Lower panel: Ground state energy relative to the nonrotating ground state, $[E(L)-E(0)] / E_{0}$, where $E_{0}=\hbar^{2} / m a_{\mathrm{dd}}^{2}$, as a function of angular momentum for values of $\varepsilon_{\mathrm{dd}}$ as given in the legend (the numbers given apply to the lines from top to bottom). The corresponding superfluid fractions $f_{s}$ at zero angular momentum for these values are 1.0, 1.0, 0.81, 0.67, 0.46, and 0.30. The insets in the lower panel show the phase plots in the ring plane (at $z=0$ ), with superimposed density contours (white lines), for $\varepsilon_{\mathrm{dd}}=$ 1.95 at $L / N \hbar=1.0$ (upper inset) and $\varepsilon_{\mathrm{dd}}=2.03$ at $L / N \hbar=0.3$ and 0.74 (lower insets). (Note the different color/ gray map scale.)

capable of supporting a persistent current. Complementary to the data shown in Fig. 2, in Fig. 3 we also present the angular momentum of the ground state in the rotating frame as a function of the rotation frequency $\Omega$. In the superfluid regime, the angular momentum displays its familiar step function behavior with the characteristic jump from $L / N \hbar=0$ to 1 at a critical frequency $\Omega_{\text {crit }}$ upon localization of the vortex at the torus center. When the system is in the supersolid regime, however, it also shows a linear gain in angular momentum with increased $\Omega$, reflecting its solid-body response to the rotation. Note that the parameters chosen here are representative for the general behavior of the system, as confirmed by the analytic approach developed below. 


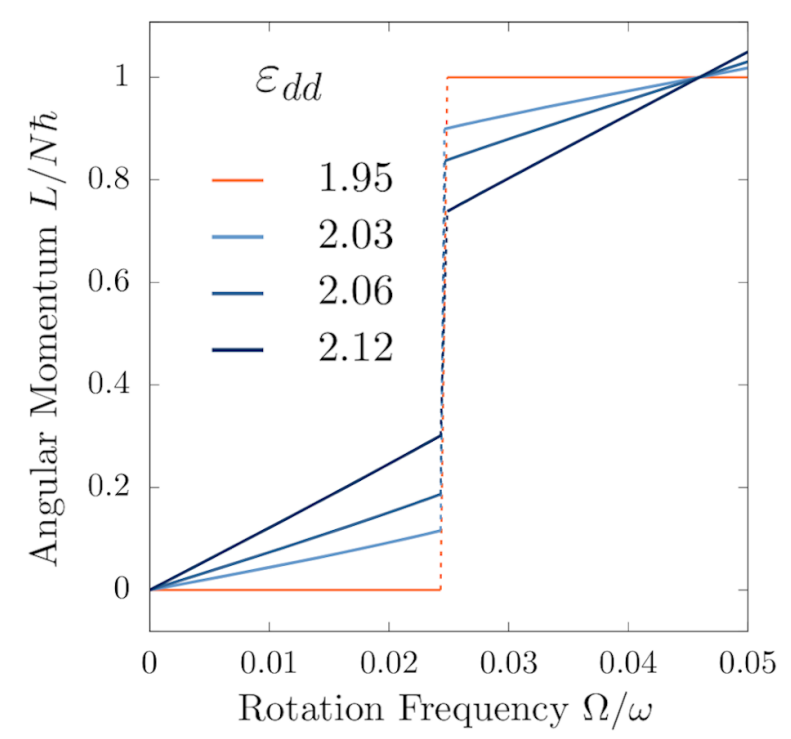

FIG. 3. Angular momentum $L / N \hbar$ of the ground state in the rotating frame as a function of the ratio $\Omega / \omega$ for given values of $\varepsilon_{\mathrm{dd}}$. In the superfluid phase for $\varepsilon_{\mathrm{dd}}=1.95$ one observes the typical jump from $L / N \hbar=0$ to 1 (marked by the dashed line).

\section{ANALYTICAL RESULTS}

To understand the results in the supersolid phase we adopt a two-component picture and model the condensate as $f_{s} N$ particles acting as a superfluid, and $\left(1-f_{s}\right) N$ particles acting as a classical solid. Considering the energy as a function of angular momentum, the two lowest energetic branches must then for low angular momenta correspond to classical rotation in the solid part, plus either a single or no vortex in the superfluid. For the branch without a vortex the energy relevant to the angular momentum considerations here is thus $E_{1}=$ $L^{2} / 2 I_{\text {solid }}$, where $I_{\text {solid }}=\left(1-f_{s}\right) N m \rho_{0}^{2}$ (using $\left.\left\langle\rho^{2}\right\rangle \approx \rho_{0}^{2}\right)$ is the approximate moment of inertia of the solid component. Similarly, the energy for the single-vortex branch is $E_{2}=$ $\left(L-f_{s} N \hbar\right)^{2} / 2 I_{\text {solid }}+E_{V}$, where $E_{V}$ is the energy difference between the states with zero and one vortex for a superfluid with $f_{s} N$ particles. In order to estimate this energy we make the following ansatz for the order parameter of a superfluid with $f_{s} N$ particles

$$
\psi_{\mathrm{sf}}(\mathbf{r})=\sqrt{\frac{f_{s} N m \omega \sqrt{\lambda}}{2 \pi^{2} \rho_{0} \hbar}} e^{-m \omega\left(\rho-\rho_{0}\right)^{2} / 2 \hbar} e^{-m \lambda \omega z^{2} / 2 \hbar} e^{i \ell_{\mathrm{sf}} \varphi},
$$

where $\ell_{\mathrm{sf}}$ the dimensionless angular momentum per particle in the superfluid. This is an approximate solution to the Schrödinger equation with the Hamiltonian

$$
H=-\frac{\hbar^{2} \nabla^{2}}{2 m}+\frac{m \omega^{2}}{2}\left[\left(\rho-\rho_{0}\right)^{2}+\lambda^{2} z^{2}\right]
$$

in the limit $\rho_{0} \gg \sqrt{\hbar / m \omega}$, corresponding to the neglect of the bending of the torus (as is the case in, for example, Refs. [75,76]). One may also consider a straight tube in Cartesian coordinates, confined harmonically in two directions with periodic boundary conditions in the third direction. This ansatz can thus be seen as a decent approximation for the weakly interacting condensate when the bending of the torus is small compared to the harmonic length. We further assume that the density is the same in the states with and without a vortex (which is reasonable for the ring geometry and also corroborated by our numerical data). The vortex energy is then estimated to be

$$
E_{V}=\frac{f_{s} N \hbar^{2}}{2 m \rho_{0}^{2}},
$$

which implies that the two energetic branches intersect at $L / N \hbar=1 / 2$, i.e., the ground state in the supersolid phase always changes between zero and one vortex at this value. This in turn means that $E(L)$ has a local minimum at $L / N \hbar=$ $f_{s}$ whenever $f_{s}>1 / 2$, and there can thus exist a persistent current also when the BEC is in the supersolid phase, which agrees well with the numerical data shown in Fig. 2. With this interpretation the energetic barrier that prevents the decay of the vortex state consists of two parts: One with just solid-body rotation (to the left of the local maximum), and the other with a single vortex plus solid-body rotation in the reverse direction (to the right of the local maximum). These peculiar counterrotating states are, however, not rotational ground states of the system, as adding the energy term $-\Omega L$ never puts them lower in energy than the single-vortex state with no solid-body rotation. While the intersection of the two aforementioned energy branches is the same under rotation, the locations and values of the minima are changed. By comparing the two energies in the rotating frame, we find the critical value for the rotation frequency, where the minimum of the first-vortex branch becomes lower than the one without a vortex, to be

$$
\Omega_{\text {crit }}=\frac{\hbar}{2 m \rho_{0}^{2}} \text {. }
$$

This critical frequency is independent of the superfluid fraction, in agreement with the data shown in Fig. 3. The value predicted by our model $\Omega_{\text {crit }} / \omega \approx 0.03$ overestimates the critical frequency compared with the numerical results due to a deviation from the approximation $\left\langle\rho^{2}\right\rangle \approx \rho_{0}^{2}$ for our particular parameters. Although states with a single vortex become energetically favorable for rotations beyond the critical frequency (5), there is still a barrier separating the states with and without a vortex. If the condensate starts in a state with no vortex and the rotation frequency is gradually increased, this barrier will disappear when $d\left(E_{1}-\Omega L\right) / d L=0$ at $L / N \hbar=1 / 2$, i.e., when $\Omega=\Omega_{\text {crit }} /\left(1-f_{s}\right)$. If we instead start from the vortex state and decrease the rotation frequency, this barrier does not completely vanish for $f_{s}>1 / 2$. On the other hand, for $f_{s}<1 / 2$, the barrier will disappear when $d\left(E_{2}-\Omega L\right) / d L=$ 0 at $L / N \hbar=1 / 2$, which occurs at $\Omega=\Omega_{\text {crit }}\left(1-2 f_{s}\right) /(1-$ $f_{s}$ ). These results give rise to hysteretic behavior in the supersolid phase, which can be seen by considering a setting where energy and angular momentum are not conserved (i.e., if there is dissipation, as would be realistic in an experimental setting). Starting by increasing the rotation frequency from zero the system then first takes angular momentum classically according to $L=I_{\text {solid }} \Omega$. When the rotation frequency reaches $\Omega=\Omega_{\text {crit }}$, a single vortex is energetically favorable but separated by an energy barrier, i.e., the state with just solid-body rotation is metastable against the first vortex. This metastability persists up until $\Omega=\Omega_{\text {crit }} /\left(1-f_{s}\right)$, where the barrier disappears and the first vortex can enter the superfluid compo- 


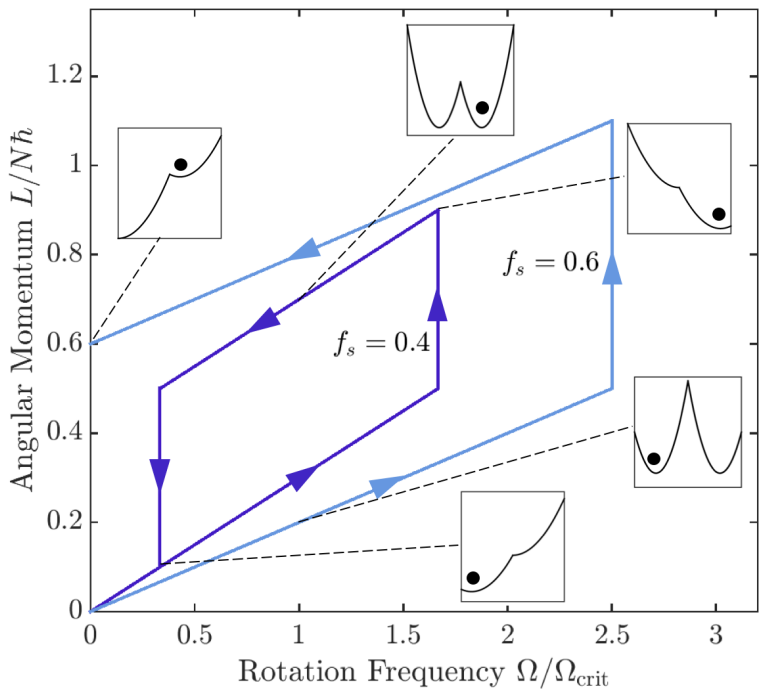

FIG. 4. Schematic illustration of the change in angular momentum as the rotation frequency changes for two different values of the superfluid fraction, $f_{s}=0.4$ (dark blue/gray line) and $f_{s}=0.6$ (light blue/gray line), chosen on either side of $f_{s}=1 / 2$. The arrows indicate the increase and decrease in $\Omega$, respectively. The insets qualitatively show the ground state energy as a function of angular momentum in the rotating frame, at rotation frequencies as indicated by the dashed lines. The bullet in each inset marks the current rotational ground state.

nent. As $\Omega$ is instead decreased, we have two different types of trajectories depending on the value of the superfluid fraction. For $f_{s}<1 / 2$ the condensate loses angular momentum continuously until the vortex leaves at $\Omega=\Omega_{\text {crit }}\left(1-2 f_{s}\right) /\left(1-f_{s}\right)$, i.e., there is hysteresis. For $f_{s}>1 / 2$, the vortex does not leave the condensate since the energetic barrier as mentioned never disappears. Consequently, the condensate only loses angular momentum corresponding to solid-body rotation. This cycle of bistability is illustrated in Fig. 4.

Finally, we wish to address the numerical results where it was observed that condensates with $f_{s}=1$ at $L=0$ could enter a density-modulated phase by forcing them to take angular momentum; see Fig. 2 for $\varepsilon_{\mathrm{dd}}=1.95$. We investigate this phenomenon by considering the different ways the condensate can take angular momentum while simultaneously being allowed to change its superfluid fraction, i.e., by letting $f_{s}$ be a function of angular momentum. We make the assumption that at an energy cost $\left(1-f_{s}\right) \Delta$ the condensate may change such that a fraction $\left(1-f_{s}\right)$ of its particles can take angular momentum in a solid-body manner instead of a superfluid one. In other words, the condensate is allowed to pay a price to be able to add angular momentum in potentially cheaper way. At low angular momenta the relevant energies per particle are then

$$
\epsilon_{1}(\ell)=\tilde{\epsilon}_{V}(\ell), \quad \epsilon_{2}(\ell)=\left(1-f_{s}\right) \Delta+\frac{\hbar^{2} \ell^{2}}{2\left(1-f_{s}\right) I},
$$

where $\ell=\ell / N \hbar, I=m \rho_{0}^{2}$, and $\tilde{\epsilon}_{V}(\ell)$ the usual positive curvature energy associated with vortex entry in a superfluid, satisfying $\tilde{\epsilon}(0)=0$ and $\tilde{\epsilon}(1)=\epsilon_{V}$ where $\epsilon_{V}=\hbar^{2} /\left(2 m \rho_{0}^{2}\right)$. By letting $\epsilon_{2}$ be a functional of $f_{s}(\ell)$ and considering its first variation, it is found that the functional form

$$
f_{s}(\ell)=1-\ell \sqrt{\frac{\epsilon_{V}}{\Delta}}
$$

minimizes the energy. This result implies

$$
\epsilon_{2}(\ell)=2 \ell \sqrt{\epsilon_{V} \Delta}
$$

that is, this branch has a linear increase in energy with angular momentum as opposed to the one with positive curvature. Whenever

$$
2 \sqrt{\epsilon_{V} \Delta}<\left.\frac{d \tilde{\epsilon}_{V}}{d \ell}\right|_{\ell=0}
$$

it is thus energetically favorable for the superfluid fraction to go below unity at low angular momenta, and density modulations will appear. We can apply the same reasoning for angular momenta close to and lower than $\ell=1$. Instead of following the vortex trajectory corresponding to the energy $\tilde{\epsilon}_{V}(\ell)$ described above, we imagine that the condensate may lower its angular momentum from $\ell=1$ by keeping a centered vortex in the superfluid component but at a lowered superfluid fraction. The total angular momentum can then be lowered further by adding angular momentum to the resulting solid component in a counterrotating manner. The relevant competing energies are then

$$
\begin{aligned}
& \epsilon_{1}(\ell)=\tilde{\epsilon}_{V}(\ell), \\
& \epsilon_{3}(\ell)=\left(1-f_{s}\right) \Delta+f_{s} \frac{\hbar^{2}}{2 I}+\frac{\hbar^{2}\left(\ell-f_{s}\right)^{2}}{2\left(1-f_{s}\right) I},
\end{aligned}
$$

to which we in a similar manner as before find the optimal superfluid fraction

$$
f_{s}(\ell)=1-(1-\ell) \sqrt{\frac{\epsilon_{V}}{\Delta}},
$$

which leads to the energy

$$
\epsilon_{3}(\ell)=\epsilon_{V}+2(1-\ell)\left(\sqrt{\epsilon_{V} \Delta}-\epsilon_{V}\right) .
$$

Expressing $\Delta$ in units of the vortex energy according to $\Delta=$ $\xi^{2} \epsilon_{V}$ where $\xi>0$ is dimensionless, the relevant energies can be summarized as

$$
\begin{aligned}
& \epsilon_{1}(\ell)=\tilde{\epsilon}_{V}(\ell), \quad \epsilon_{2}(\ell)=2 \xi \epsilon_{V} \ell, \\
& \epsilon_{3}(\ell)=\epsilon_{V}+2(\xi-1) \epsilon_{V}(1-\ell) .
\end{aligned}
$$

Since $\epsilon_{2}$ and $\epsilon_{3}$ correspond to physical systems with $f_{s}<1$, this shows that it is possible for the condensate with $f_{s}=1$ at $\ell=0$ to enter the supersolid phase as angular momentum is added to it, even when $\varepsilon_{\mathrm{dd}}$ is lower than its critical value. The energy dependence on angular momentum should according to these results be either a linear increase close to $\ell=0$ or a linear decrease (for $\xi>1$ ) close to $\ell=1$, where the slope for the linear increase is greater than for the decreasing one. This is in accordance to our numerical data, which can be seen in Fig. 2. We have observed this behavior in general as long as $\varepsilon_{\mathrm{dd}}$ is not too close to its critical value. As the relative interaction strength nears this critical point, the previously linear slopes take on a positive curvature similar to the results for condensates that have $f_{s}<1$ at $\ell=0$. This suggests that the assumption where the angular momentum behavior of a 
particle can be changed at a constant energy cost $\Delta$ is no longer a good one.

\section{CONCLUSIONS}

In conclusion, we have seen that a toroidal dipolar Bose gas may support a persistent current also in the supersolid phase, however, with a behavior qualitatively different from the superfluid phase. Additionally, we have found that the condensate may transition between the superfluid and the supersolid phase simply by adding angular momentum to it. The associated minimum in the rotation energy gives rise to hysteretic behavior, similarly to what has been observed in toroidally confined superfluid sodium BECs [68]. We expect that these properties of supersolids in ring-shaped traps are accessible in current experiments with gases of dysprosium or erbium.

\section{ACKNOWLEDGMENTS}

This work was financially supported by the Knut and Alice Wallenberg Foundation, the Swedish Research Council, and NanoLund. Communications with G. M. Kavoulakis and P. Stürmer are acknowledged.
[1] E. P. Gross, Phys. Rev. 106, 161 (1957); Ann. Phys. 4, 57 (1958).

[2] C. N. Yang, Rev. Mod. Phys. 34, 694 (1962).

[3] A. Andreev and I. Lifshits, Zh. Eksp. Teor. Fiz. 56, 2057 (1969) [JETP 29, 1107 (1969)].

[4] G. V. Chester, Phys. Rev. A 2, 256 (1970).

[5] A. J. Leggett, Phys. Rev. Lett. 25, 1543 (1970).

[6] E. Kim and M. H. W. Chan, Nature (London) 427, 225 (2004); Science 305, 1941 (2004).

[7] S. Balibar, Nature (London) 464, 176 (2010).

[8] M. Boninsegni and N. V. Prokof'ev, Rev. Mod. Phys. 84, 759 (2012).

[9] M. H. W. Chan, R. B. Hallock, and L. Reatto, J. Low Temp. Phys. 172, 317 (2013).

[10] D. Y. Kim and M. H. W. Chan, Phys. Rev. Lett. 109, 155301 (2012).

[11] Y. Pomeau and S. Rica, Phys. Rev. Lett. 72, 2426 (1994).

[12] N. Henkel, R. Nath, and T. Pohl, Phys. Rev. Lett. 104, 195302 (2010).

[13] F. Cinti, P. Jain, M. Boninsegni, A. Micheli, P. Zoller, and G. Pupillo, Phys. Rev. Lett. 105, 135301 (2010).

[14] S. Saccani, S. Moroni, and M. Boninsegni, Phys. Rev. B 83, 092506 (2011).

[15] J. Léonard, A. Morales, P. Zupancic, T. Donner, and T. Esslinger, Science 358, 1415 (2017); J. Léonard, A. Morales, P. Zupancic, T. Esslinger, and T. Donner, Nature (London) 543, 87 (2017).

[16] Y. J. Lin, K. Jiménez-García, and I. B. Spielman, Nature (London) 471, 83 (2011).

[17] J. Li, W. Huang, B. Shteynas, S. Burchesky, F. C. Top, E. Su, J. Lee, A. O. Jamison, and W. Ketterle, Phys. Rev. Lett. 117, 185301 (2016).

[18] J.-R. Li, J. Lee, W. Huang, S. Burchesky, B. Shteynas, F. C. T. Top, A. O. Jamison, and W. Ketterle, Nature (London) 543, 91 (2017).

[19] T. Lahaye, C. Menotti, L. Santos, M. Lewenstein, and T. Pfau, Rep. Prog. Phys. 72, 126401 (2009).

[20] M. A. Baranov, M. Dalmonte, G. Pupillo, and P. Zoller, Chem. Rev. 112, 5012 (2012).

[21] F. Böttcher, J.-N. Schmidt, M. Wenzel, J. Hertkorn, M. Guo, T. Langen, and T. Pfau, Phys. Rev. X 9, 011051 (2019).

[22] L. Tanzi, E. Lucioni, F. Famà, J. Catani, A. Fioretti, C. Gabbanini, R. N. Bisset, L. Santos, and G. Modugno, Phys. Rev. Lett. 122, 130405 (2019).
[23] L. Chomaz, D. Petter, P. Ilzhöfer, G. Natale, A. Trautmann, C. Politi, G. Durastante, R. M. W. van Bijnen, A. Patscheider, M. Sohmen et al., Phys. Rev. X 9, 021012 (2019).

[24] G. Natale, R. M. W. van Bijnen, A. Patscheider, D. Petter, M. J. Mark, L. Chomaz, and F. Ferlaino, Phys. Rev. Lett. 123, 050402 (2019).

[25] L. Tanzi, S. M. Roccuzzo, E. Lucioni, F. Famà, A. Fioretti, C. Gabbanini, G. Modugno, A. Recati, and S. Stringari, Nature (London) 574, 382 (2019).

[26] M. Guo, F. Böttcher, J. Hertkorn, J.-N. Schmidt, M. Wenzel, H. P. Büchler, T. Langen, and T. Pfau, Nature (London) 574, 386 (2019).

[27] J. Hertkorn, F. Böttcher, M. Guo, J. N. Schmidt, T. Langen, H. P. Büchler, and T. Pfau, Phys. Rev. Lett. 123, 193002 (2019).

[28] H. Kadau, M. Schmitt, M. Wenzel, C. Wink, T. Maier, I. FerrierBarbut, and T. Pfau, Nature (London) 530, 194 (2016).

[29] M. Schmitt, M. Wenzel, F. Böttcher, I. Ferrier-Barbut, and T. Pfau, Nature (London) 539, 259 (2016).

[30] I. Ferrier-Barbut, H. Kadau, M. Schmitt, M. Wenzel, and T. Pfau, Phys. Rev. Lett. 116, 215301 (2016).

[31] L. Chomaz, S. Baier, D. Petter, M. J. Mark, F. Wächtler, L. Santos, and F. Ferlaino, Phys. Rev. X 6, 041039 (2016).

[32] M. Wenzel, F. Böttcher, T. Langen, I. Ferrier-Barbut, and T. Pfau, Phys. Rev. A 96, 053630 (2017).

[33] F. Wächtler and L. Santos, Phys. Rev. A 94, 043618 (2016); 93, 061603(R) (2016).

[34] D. Baillie, R. M. Wilson, R. N. Bisset, and P. B. Blakie, Phys. Rev. A 94, 021602(R) (2016).

[35] R. N. Bisset, R. M. Wilson, D. Baillie, and P. B. Blakie, Phys. Rev. A 94, 033619 (2016).

[36] D. Baillie and P. B. Blakie, Phys. Rev. Lett. 121, 195301 (2018).

[37] A. Bulgac, Phys. Rev. Lett. 89, 050402 (2002).

[38] D. S. Petrov, Phys. Rev. Lett. 115, 155302 (2015).

[39] D. S. Petrov and G. E. Astrakharchik, Phys. Rev. Lett. 117, 100401 (2016).

[40] G. Semeghini, G. Ferioli, L. Masi, C. Mazzinghi, L. Wolswijk, F. Minardi, M. Modugno, G. Modugno, M. Inguscio, and M. Fattori, Phys. Rev. Lett. 120, 235301 (2018).

[41] C. Cabrera, L. Tanzi, J. Sanz, B. Naylor, P. Thomas, P. Cheiney, and L. Tarruell, Science 359, 301 (2018).

[42] T. D. Lee, K. Huang, and C. N. Yang, Phys. Rev. 106, 1135 (1957). 
[43] A. R. P. Lima and A. Pelster, Phys. Rev. A 84, 041604(R) (2011); 86, 063609 (2012).

[44] L. Chomaz, R. M. W. van Bijnen, D. Petter, G. Faraoni, S. Baier, J. H. Becher, M. J. Mark, F. Wächtler, L. Santos, and F. Ferlaino, Nat. Phys. 14, 442 (2018).

[45] D. Petter, G. Natale, R. M. W. van Bijnen, A. Patscheider, M. J. Mark, L. Chomaz, and F. Ferlaino, Phys. Rev. Lett. 122, 183401 (2019).

[46] Y. Kora and M. Boninsegni, J. Low Temp. Phys. 197, 337 (2019).

[47] D. Petter, A. Patscheider, G. Natale, M. J. Mark, M. A. Baranov, R. v. Bijnen, S. M. Roccuzzo, A. Recati, B. Blakie, D. Baillie, L. Chomaz, and F. Ferlaino, arXiv:2005.02213 [cond-mat.quantgas] (2020).

[48] A. J. Leggett, J. Stat. Phys. 93, 927 (1998).

[49] S. M. Roccuzzo, A. Gallemí, A. Recati, and S. Stringari, Phys. Rev. Lett. 124, 045702 (2020); A. Gallemí, S. M. Roccuzzo, S. Stringari, and A. Recati, Phys. Rev. A 102, 023322 (2020).

[50] F. Ancilotto, M. Barranco, M. Pi, and L. Reatto, arXiv:2002.05934 [cond-mat.quant-gas] (2020).

[51] F. Bloch, Phys. Rev. A 7, 2187 (1973).

[52] K. Kärkkäinen, J. Christensson, G. Reinisch, G. M. Kavoulakis, and S. M. Reimann, Phys. Rev. A 76, 043627 (2007).

[53] S. Bargi, F. Malet, G. M. Kavoulakis, and S. M. Reimann, Phys. Rev. A 82, 043631 (2010).

[54] M. Cominotti, D. Rossini, M. Rizzi, F. Hekking, and A. Minguzzi, Phys. Rev. Lett. 113, 025301 (2014).

[55] A. Muñoz Mateo, A. Gallemí, M. Guilleumas, and R. Mayol, Phys. Rev. A 91, 063625 (2015).

[56] A. Roussou, G. D. Tsibidis, J. Smyrnakis, M. Magiropoulos, N. K. Efremidis, A. D. Jackson, and G. M. Kavoulakis, Phys. Rev. A 91, 023613 (2015).

[57] J. Polo, R. Dubessy, P. Pedri, H. Perrin, and A. Minguzzi, Phys. Rev. Lett. 123, 195301 (2019).

[58] L. Amico, A. Osterloh, and F. Cataliotti, Phys. Rev. Lett. 95, 063201 (2005).
[59] D. Aghamalyan, M. Cominotti, M. Rizzi, D. Rossini, F. Hekking, A. Minguzzi, L.-C. Kwek, and L. Amico, New J. Phys. 17, 045023 (2015).

[60] M. Abad, M. Guilleumas, R. Mayol, M. Pi, and D. M. Jezek, Phys. Rev. A 81, 043619 (2010).

[61] M. Abad, M. Guilleumas, R. Mayol, M. Pi, and D. M. Jezek, Europhys. Lett. 94, 10004 (2011).

[62] F. Malet, G. M. Kavoulakis, and S. M. Reimann, Phys. Rev. A 84, 043626 (2011).

[63] S. K. Adhikari, Phys. Rev. A 85, 053631 (2012).

[64] E. O. Karabulut, F. Malet, G. M. Kavoulakis, and S. M. Reimann, Phys. Rev. A 87, 033615 (2013).

[65] X.-F. Zhang, W. Han, L. Wen, P. Zhang, R.-F. Dong, H. Chang, and S.-G. Zhang, Sci. Rep. 5, 8684 (2015).

[66] M. Kunimi and Y. Kato, Phys. Rev. B 86, 060510(R) (2012).

[67] K. C. Wright, R. B. Blakestad, C. J. Lobb, W. D. Phillips, and G. K. Campbell, Phys. Rev. Lett. 110, 025302 (2013).

[68] S. Eckel, J. G. Lee, F. Jendrzejewski, N. Murray, C. W. Clark, C. J. Lobb, W. D. Phillips, M. Edwards, and G. K. Campbell, Nature (London) 506, 200 (2014).

[69] S. Eckel, A. Kumar, T. Jacobson, I. B. Spielman, and G. K. Campbell, Phys. Rev. X 8, 021021 (2018).

[70] L. Amico, G. Birkl, M. Boshier, and L.-C. Kwek, New J. Phys. 19, 020201 (2017).

[71] H. Saito, J. Phys. Soc. Jpn. 85, 053001 (2016).

[72] S. M. Roccuzzo and F. Ancilotto, Phys. Rev. A 99, 041601(R) (2019).

[73] P. B. Blakie, D. Baillie, L. Chomaz, and F. Ferlaino, Phys. Rev. Res. 2, 043318 (2020).

[74] D. A. Butts and D. S. Rokhsar, Nature (London) 397, 327 (1999).

[75] O. Morizot, Y. Colombe, V. Lorent, H. Perrin, and B. M. Garraway, Phys. Rev. A 74, 023617 (2006).

[76] W. H. Heathcote, E. Nugent, B. T. Sheard, and C. J. Foot, New J. Phys. 10, 043012 (2008). 